\title{
Electronic lifetimes in ballistic quantum dots electrostatically coupled to metallic environments
}

\author{
Francisco Guinea ${ }^{1}$, Rodolfo A. Jalabert ${ }^{2}$, and Fernando Sols $^{3}$ \\ 1 Instituto de Ciencias Materiales, CSIC, Campus de Cantoblanco, E-28043 Madrid, Spain \\ 2 Institut de Physique et Chimie des Matériaux de Strasbourg, \\ UMR 7504 (CNRS-ULP) 23 rue du Loess, BP 4367034 Strasbourg Cedex 2, France \\ 3 Departamento de Física Teórica de la Materia Condensada e Instituto "Nicolás Cabrera" \\ Universidad Autónoma de Madrid, Cantoblanco, E-28043 Madrid, Spain
}

\begin{abstract}
We calculate the lifetime of low-energy electronic excitations in a two-dimensional quantum dot near a metallic gate. We find different behaviors depending on the relative values of the dot size, the dot-gate distance and the Thomas-Fermi screening length within the dot. The standard Fermi liquid behavior is obtained when the dot-gate distance is much shorter than the dot size or when it is so large that intrinsic effects dominate. Departures from the Fermi liquid behavior are found in the unscreened dipole case of small dots far away from the gate, for which a Caldeira-Leggett model is applicable. At intermediate distances, a marginal Fermi liquid is obtained if there is sufficient screening within the dot. In these last two non-trivial cases, the level width decays as a power law with the dot-gate distance.
\end{abstract}

PACS numbers: 73.21.-b, 73.22-f, 73.23.-b

\section{INTRODUCTION.}

The understanding of lifetimes of electronic states in finite low-dimensional devices presents subtleties not found in extended systems 1.2 .3 .4 . The lifetime of quasiparticle excitations (with energy $E$ ) in bulk three- and two-dimensional clean electron systems scales as $(E-$ $\left.E_{F}\right)^{2}$, for $E$ sufficiently close to the Fermi energy, $E_{F} \underset{5,6}{ }$. Such a behavior results from the combined effects of the screened electron-electron interaction and the continuous spectral density provided by the internal electronic environment (Fermi sea). The above energy dependence can be readily obtained from a Fermi Golden Rule (FGR) approach using the matrix elements of the effective interaction expressed in terms of the polarizability of the metal ${ }^{5}$. This response function can also be viewed as resulting from an external environment.

The corrections induced by disorder and finite size can be systematically computed using an expansion in the inverse conductance, $g^{-1}=\Delta_{0} / E_{c}$, where $\Delta_{0}$ is the typical level spacing, and $E_{c}=\hbar D / L^{2}$ is the Thouless en$\operatorname{ergy}(D$ is the diffusion coefficient, and $L$ the size of the system) $)^{7.8}$. The application of a FGR approach for intrinsic quasiparticle decay is valid for sizes (or quasiparticle energies) large enough that the system can be described as an environment with a continuous spectrum ${ }^{3.4}$. On the other hand, in very small dots, it is the presence of nearby metallic gates that ensures the existence of decay channels at arbitrarily low energies. This effect becomes of crucial importance in determining the width of the low lying electronic excitations 9.10 .

The purpose of the present work is to investigate the quasiparticle lifetimes in quantum dots of various sizes in the vicinity of an extended metallic gate. In this way, we can study the crossover between the regimes in which the external environment does not introduce qualitative changes with respect to the case of the isolated dot, and that in which significant departures from Fermi liquid behavior are obtained. We consider the simple geometry sketched in Fig. 1 with a two-dimensional dot of lateral size $L$, at a distance $z$ from the gate. Another important scale is the the Thomas-Fermi screening length for the dot, $\lambda_{\mathrm{TF}}=\pi \hbar^{2} \epsilon_{0} / m^{*} e^{2}\left(m^{*}\right.$ is the electronic effective mass and $\epsilon_{0}$ the dielectric constant). The metallic gate will be described as an Ohmic environment, that is, as one with a low-energy spectral density proportional to the frequency 11 . The different behaviors mentioned above are obtained by varying the relative values of the length scales $L, z$, and $\lambda_{\mathrm{TF}}$.

The widely used Caldeira-Leggett model, understood as that with Ohmic spectral density and coupling linear in the particle coordinate $\frac{12}{2}$, corresponds to the limiting case $z / L, \lambda_{\mathrm{TF}} / L \gg 1$. This is the regime which exhibits the largest departure from the Fermi liquid behavior, a result which is consistent with the decay of persistent currents predicted for mesoscopic rings where the electronic dipoles couple to an Ohmic oscillator bath ${ }^{13}$. Clarifying the applicability and physical consequences of different environments is one of the guiding lines of this work.

The question of electron decay is related to the loss of phase coherence suffered by electrons in a dot, a problem which has been the object of experimental attention 14.15 .16 .17 . Here we propose a framework within which one can study a variety of geometrical setups where extrinsic mechanisms compete with the intrinsic ones to cause electron wave decoherence.

The next section introduces the model considered in this work. Then, we present calculations for the case $z / L, \lambda_{\mathrm{TF}} / L \gg 1$. Section IV discusses the regime $z / L \ll$ 1 , while in Sec. $\nabla$ we analyze the situation when $z / L \gg$ 1 and $\lambda_{\mathrm{TF}} / L \ll 1$. The main conclusions, as well as the experimental relevance of our work, are discussed in Sec. VI. 


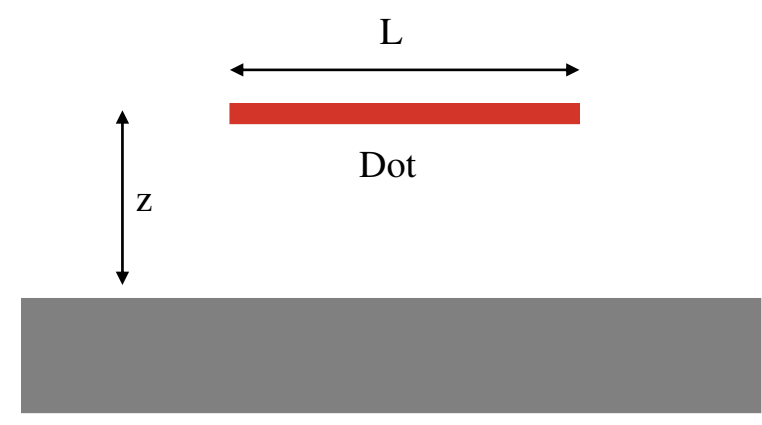

Gate

\section{THE MODEL.}

As described in the introduction, our system is given by a dot coupled to a metallic gate (see Fig. (1). Such an electrostatic interaction is governed by the Hamiltonian

$$
\mathcal{H}_{\text {int }}=\int_{A} \hat{V}_{z}(\mathbf{r}) \hat{\rho}(\mathbf{r}) d \mathbf{r},
$$

where $A=L^{2}$ is the area of the dot, $\mathbf{r}$ is a twodimensional vector in the plane of the dot, $\hat{\rho}(\mathbf{r})$ is the operator describing the local electronic density fluctuations of the dot, and $\hat{V}_{z}(\mathbf{r})$ represents the potential induced on the dot by the gate (with $z$ the separation dot-gate).

The electronic states of the dot in the absence of the gate will be assumed to be well described by an independent electron analysis. The consistency of this approximation in the various regimes will be discussed in the sequel. Using the FGR the probability per unit time of a transition between the electronic states $n$ and $m$ (transition rate) is:

$\Gamma_{m n}=\int_{A} d \mathbf{r} \int_{A} d \mathbf{r}^{\prime}\langle m|\hat{\rho}(\mathbf{r})| n\rangle\left\langle n\left|\hat{\rho}\left(\mathbf{r}^{\prime}\right)\right| m\right\rangle S\left(\mathbf{r}, \mathbf{r}^{\prime}, z ; E_{n}-E_{m}\right)$,

where the structure factor

$$
S\left(\mathbf{r}, \mathbf{r}^{\prime}, z ; \Delta E\right)=\int \frac{d t}{\hbar} e^{i \Delta E t / \hbar}\left\langle\hat{V}_{z}\left(\mathbf{r}^{\prime}, t\right) \hat{V}_{z}(\mathbf{r}, 0)\right\rangle
$$

describes the fluctuations of the metallic environment as experienced in the quantum dot. The averages are taken with respect to the degrees of freedom of the environment. The second order processes involved in the calculation of the transition rates of Eq. (21) are represented by the diagram shown in Fig. 2

Within the one-particle picture that we adopted for the $\operatorname{dot}\langle m|\hat{\rho}(\mathbf{r})| n\rangle=\Psi_{m}^{*}(\mathbf{r}) \Psi_{n}(\mathbf{r})$, where $\Psi_{n}$ is the wave function of the effectively independent electron, and Eq. (2) can be written as

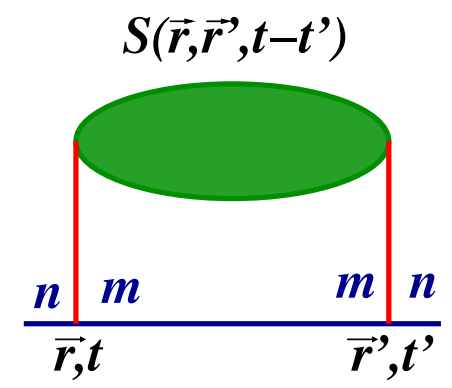

FIG. 2: Diagram representing the calculation in Eq. (2). The filled bubble describes the time-dependent response of the environment (improper polarization). Labels $m$ and $n$ stand for electron states within the dot.

$$
\Gamma_{m n}=\int \frac{d^{2} q}{(2 \pi)^{2}}\left|\left\langle m\left|e^{i \mathbf{q} \cdot \mathbf{r}}\right| n\right\rangle\right|^{2} S(\mathbf{q}, z ; \Delta E),
$$

where $S(\mathbf{q}, z, \Delta E)$ is the Fourier transform of the structure factor.

Notice that now a quasi-particle does not decay into a two-particle-one-hole configuration (like in the case of intrinsic decay), but into another one-particle state. The use of FGR is then justified by the large density of final states provided by the environment (metallic gate).

The properties of the environment determine the precise form of the bubble in Fig. 2] However, we can infer some general features fulfilled by the physically relevant environments we are interested in:

(i) An Ohmic environment implies that, at low energies,

$$
S\left(\mathbf{r}, \mathbf{r}^{\prime}, z ; \Delta E\right) \simeq|\Delta E| \tilde{S}\left(\mathbf{r}, \mathbf{r}^{\prime}, z\right) ;
$$

(ii) For small $|\mathbf{r}|$ and $\left|\mathbf{r}^{\prime}\right|$ we will always have, effectively,

$$
\tilde{S}\left(\mathbf{r}, \mathbf{r}^{\prime}, z\right) \simeq \mathbf{r} \overleftrightarrow{S_{0}}(z) \mathbf{r}^{\prime}
$$

(iii) $\tilde{S}\left(\mathbf{r}, \mathbf{r}^{\prime}, z\right) \rightarrow 0$ for $\left|\mathbf{r}-\mathbf{r}^{\prime}\right|$ sufficiently large with respect to the characteristic length scales of the environment and the dot-gate coupling. These general properties still allow for different regimes, which will be thoroughly discussed in the following chapters.

The lifetime of a state $n$ is obtained by considering all the possible transitions to lower states above the Fermi energy:

$$
\Gamma_{n}=\sum_{E_{F}<E_{m}<E_{n}} \Gamma_{m n} .
$$

We are interested in describing the energy dependence of the level width. Therefore, we will average over nearby eigenstates: 


$$
\Gamma(E)=\frac{1}{\nu(E)} \sum_{n} \Gamma_{n} \delta_{\epsilon}\left(E-E_{n}\right)
$$

where $\delta_{\epsilon}$ represents a smoothed $\delta$ function of width $\epsilon$ (which we take as an energy scale of the order of a few level spacings) and $\nu(E) \equiv \sum_{n} \delta_{\epsilon}\left(E-E_{n}\right)$ is the smoothed density of states within the dot.

\section{DIPOLAR APPROXIMATION.}

For dots far away from the gate $(z \gg L)$ and $L$ so small that internal screening effects can be neglected $(L \ll$
$\left.\lambda_{\mathrm{TF}}\right)$, the electric field penetrates the dot and, in the low-frequency limit, we can use the dipolar expansion represented in Eq. (6). For simplicity, we will take the

tensor $\overleftrightarrow{S_{0}}$ as diagonal and isotropic, $\overleftrightarrow{S_{0}} \simeq S_{0}(z)$. Relaxing this assumption would not lead to qualitative changes in the results. An estimation of $S_{0}(z)$ in a simple situation is given in Appendix A.

$$
\Gamma_{m n}=S_{0}(z)\left(E_{n}-E_{m}\right) \int d \mathbf{r} d \mathbf{r}^{\prime} \Psi_{n}^{*}(\mathbf{r}) \Psi_{m}\left(\mathbf{r}^{\prime}\right) \mathbf{r} \cdot \mathbf{r}^{\prime} \Psi_{m}(\mathbf{r}) \Psi_{n}^{*}\left(\mathbf{r}^{\prime}\right)=\left.S_{0}(z)\left(E_{n}-E_{m}\right)|\langle m| \mathbf{r})|n\rangle\right|^{2}
$$

The transition rate is then governed by the squared dipole matrix element. Local averages (on a scale of $\epsilon$ ) of matrix elements for an arbitrary operator $A$,

$C_{A}(E, \Delta E) \equiv \sum_{m n}|\langle m|A| n\rangle|^{2} \delta_{\epsilon}\left(E-E_{n}\right) \delta_{\epsilon}\left(\Delta E-E_{n}+E_{m}\right)$,

have been thoroughly studied $18,19,20,21$. They are interesting quantum mechanical quantities governing not only transition rates, but other experimentally revelant phenomena, like the energy absorption rate of small metallic clusters 24.25 and quantum transport in ballistic systems ${ }^{26}$. Similarly to the density of states ${ }^{22}$, $C_{A}(E, \Delta E)$ can be written as a leading smooth term plus oscillating corrections. The later are given by a periodic orbit expansion, and therefore depend on the nature of the underlying classical dynamics (i.e. chaotic vs. integrable). The smooth term is given by the Fourier transform of the classical autocorrelation function of the observable (expressed in terms of classical trajectories) and presents some scaling behavior in the case of billiard systems ${ }^{20}$. For the dipole matrix elements $(A=\mathbf{r})$ the hard wall confining potential translates into a dependence

$$
C_{\mathbf{r}}(E, \Delta E) \simeq \frac{L^{2}}{\sqrt{\nu(E)}} \frac{E^{3 / 2}}{\Delta E^{4}}
$$

for both, integrable and non-integrable systems, provided $\Delta E>\hbar v_{F} / L$. The $\Delta E^{-4}$ dependence of $C_{\mathbf{r}}(E, \Delta E)$ arises from the discontinuity of the velocity of the particle as it bounces against the hard wall. The power-law
From equations (2), (5), and (6) one can write the transition rate as of Eq. (11) is obtained from the contribution of a single bounce off the boundary. At sufficiently low frequencies, the contributions from many such bounces have to be added. This leads to interference effects and to an effective lower cutoff of the $\Delta E^{-4}$ law at the synchrotron energy $\hbar v_{F} / L$.

Like in the one-dimensional case (where the semiclassical dipole matrix element can be evaluated on general grounds ${ }^{23}$ ), the $\Delta E^{-4}$ law is preserved when we consider a soft potential, provided that $\Delta E \ll(\hbar / a) \sqrt{E / m^{*}}$, where $a$ is the length scale defining the rise of the confining potential. That is when the small energy differences that we are interested in correspond to times much larger than the collision time, and thus the detailed profile of the confining walls becomes irrelevant. For large values of $\Delta E$, the soft character of the walls comes into play, and the correlation function defined in Eq. (10) decays faster than $\Delta E^{-4}$. In the following calculations we will be using Eq. (11) for $\Delta E$ in the range $\hbar v_{F} / L<\Delta E<(\hbar / a) \sqrt{E / m^{*}}$.

In simple two-dimensional geometries, like a circular $\operatorname{disc}^{24}$ or an infinite rectangular stripe $e^{25}$, the dipole matrix element can be calculated explicitly for the unscreened case and screened (Thomas-Fermi) cases. In the calculation that follows we will only need the scaling behavior of Eq. (11) and we will not be restricted to a particular geometry.

If $\Delta_{0} \approx \hbar^{2} / m L^{2}$ is the mean level spacing within the dot, the estimation of the energy dependence of the dipole matrix elements allows us to write the level width as 


$$
\Gamma(E) \simeq \frac{S_{0}(z)}{\nu(E)} \int_{\Delta_{0}}^{E-E_{F}} d(\Delta E) \Delta E C_{\mathbf{r}}(E, \Delta E) \simeq c\left[1-\left(\frac{\hbar v_{F} / L}{E-E_{F}}\right)^{2}\right]
$$

for $E-E_{F}>\hbar v_{F} / L$, and $\Gamma(E) \simeq 0$ for $E-E_{F} \ll \hbar v_{F} / L$. The constant $c$ is discussed in the sequel. The energy dependence of the level width, depicted in Fig. 3] yields a plateau-like behavior for $\hbar v_{F} / L \ll E-E_{F} \ll E_{F}$. For $E-E_{F}$ comparable to $E_{F}$, the possible smoothing of the potential and the opening of additional relaxation mechanisms lead to a vanishing life-time, and therefore $\Gamma(E)$ increases with $E$ for $E-E_{F}>E_{F}$ (not shown in Fig. 3).

The quasiparticle decay rate for a standard Fermi liquid follows the law $\Gamma_{\mathrm{FL}}(E) \propto\left(E-E_{F}\right)^{2}$. Thus, the energy dependence obtained in Eq. (12) (a constant $\Gamma(E)$ for $\left.E-E_{F} \gg \hbar v_{F} / L\right)$ reveals a non-Fermi-liquid behavior. We have thus identified an electronic system where dissipation effects are well described by the standard (linear in the particle coordinate) Caldeira-Leggett model, the oscillator bath being formed by the quasiparticle field of the gate. Anomalous behaviors in systems described by the the Caldeira-Leggett model have been discussed in the literature ${ }^{27.28}$. Such particle-bath couplings were known to be realized in contexts where the particle coordinate (e.g. the flux through a superconducting ring) does not experience any quantum statistical constraint. Here we have shown that, under specific circumstances, such anomalous behavior can also be displayed in systems where the Pauli exclusion principle plays an essential role.

The constant $c$ in Eq. (12) depends on the distance gate-dot and on the properties of the gate (through $S_{0}(z)$ ), as well as on the details of the dot (through $C(E, \Delta E)$ and $\nu(E)$ ). Using the results of the Appendix for a two-dimensional gate, we have $c=\left(L \hbar^{2} k_{F} / m\right) z^{-2}\left(k_{F}^{\prime} l^{\prime}\right)^{-1}$, where the primes refer to parameters of the gate. For a semi-infinite threedimensional gate, the factor $z^{2} k_{F}^{\prime} l^{\prime}$ in the denominator of $S_{0}(z)$ is replaced by $z^{3} k_{F}^{\prime 2} l^{\prime}$, while for a one-dimensional gate it is replaced by $z l^{\prime}$. Thus, we find that the level width decays with the dot-gate distance as power-law, with an exponent given by the dimensionality of the gate $(d=1,2,3)$

\section{SHORT RANGE REGIME.}

For dots sufficiently close to the gate $(z \ll L)$, we expect the quasi-perfect screening provided by the gate to yield a structure factor of Eq. (5) of limited range. Let us define $\mathbf{r}^{\prime \prime}=\mathbf{r}-\mathbf{r}^{\prime}$ and assume that

$$
\bar{S} \equiv \int d \mathbf{r}^{\prime \prime} S\left(\mathbf{r}^{\prime \prime}\right)
$$

is finite. Then we may mimic the structure kernel through the approximation

$$
\tilde{S}\left(\mathbf{r}, \mathbf{r}^{\prime}, z ; \Delta E\right)=\bar{S} \Delta E \delta\left(\mathbf{r}-\mathbf{r}^{\prime}\right)
$$

for $\left|\mathbf{r}-\mathbf{r}^{\prime}\right|$ much greater than the range of $S\left(\mathbf{r}^{\prime \prime}\right)$. Let us take $\Delta E$ small enough for the density matrix element between states $m$ and $n$ with $\left(E_{n}-E_{m}\right)=\Delta E$ to be, on average, approximately independent of position and energy,

$$
\left|\Psi_{n}^{*}(\mathbf{r}) \Psi_{m}(\mathbf{r})\right|^{2} \simeq \frac{1}{A^{2}}
$$

This set of approximations leads to a transition rate from state $n$ to $m$ with the particularly simple expression:

$$
\begin{aligned}
\Gamma_{m n} & =\bar{S} \Delta E \int_{A} d \mathbf{r}\left|\Psi_{n}^{*}(\mathbf{r}) \Psi_{m}(\mathbf{r})\right|^{2} \\
& \simeq \frac{\bar{S}}{A} \Delta E .
\end{aligned}
$$

Within this regime of validity, the width of a quasiparticle state of energy $E$ can be written as

$$
\Gamma(E)=\frac{\bar{S}}{A} \nu(E) \int_{\Delta_{0}}^{E-E_{F}} d(\Delta E) \Delta E \propto\left(E-E_{F}\right)^{2},
$$

where the density of states $\nu(E)$ is assumed to be vary slowly on the scale of $E-E_{F}$ (an exact property for a two-dimensional dot).

In practice, $\bar{S}$ defined in (13) is never finite, as can be seen by noting that $\bar{S}=\lim _{\Delta E \rightarrow 0} S(\mathbf{q}=0, z, \Delta E) / \Delta E$

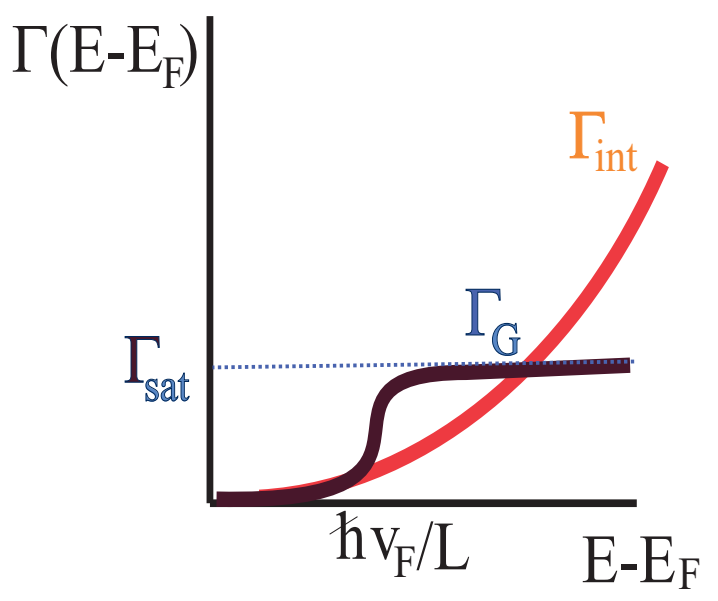

FIG. 3: Schematic behavior of the decay rate, $\Gamma(E)$, of state of energy $E$, as a function of $E$, for the unscreened dipole case discussed in Sec. III. 
and deriving $S(q, z, \Delta E)$ for any reasonable screening model (see the Appendix for specific cases). Fortunately, the requirements to obtain (17) and (18) are much less stringent than the finiteness of (13). While the electronic behavior of a dot very close to a metallic gate deserves a careful study, a preliminary investigation reveals the following results: A semi-infinite three-dimensional gate yields Fermi liquid behavior for $z \ll L$, with logarithmic corrections if it is dirty. The behavior of a dot close to a gate made of a two-dimensional electron gas is somewhat more singular. We find marginal Fermi liquid behavior $\left[\Gamma(E) \propto\left(E-E_{F}\right)\right]$ if the 2D gate is diffusive, and Fermi liquid behavior with logarithmic corrections if it is ballistic. We must note that these calculations do not include additional screening by the dot, which may be important for $L>\lambda_{\mathrm{TF}}$. For such large dots, we expect to find full Fermi liquid behavior for both two-dimensional and semi-infinite three-dimensional gates.

The result (18) has the form which corresponds to a standard Fermi liquid ${ }^{6}$. The effect of the external environment is then indistinguishable from that of the intrinsic charge fluctuations of the dot, which are not considered in the present analysis but which should dominate in the limit of very large dots. The estimation of the typical matrix element in Eq. (16) can be extended to diffusive dots 29 , leading to logarithmic corrections to Eq. (18). Similar effects for bulk diffusive systems are well known in the literature ${ }^{30}$. Those calculations ${ }^{29.30}$ have been made in the context of studies of the effect of intrinsic charge fluctuations.

\section{SCREENED DIPOLE.}

For dots far away from the gate and large enough to partially screen the external electric field $\left(z \gg L \gg \lambda_{\mathrm{TF}}\right)$ an intermediate regime should be considered. The potential which induces the electronic transitions in the dot has now to be taken as that of an external uniform electric field screened by the two-dimensional electron gas of the dot. Since the dot is two-dimensional, the screening length is independent of the electron density. In addition, in contrast with the three-dimensional case, the electric field penetrates beyond this length. Near the boundary, the charge density and the screened potential, $V_{\mathrm{TF}}(\mathbf{r})$, depend algebraically on the distance to the edge ${ }^{7.8}, \xi$, as $\xi^{-1 / 2}$. This power-law behavior allows us to estimate the dependence on the energy difference, $\Delta E$, of the transition matrix elements, to leading order in $\lambda_{\mathrm{TF}} / L$. For a ballistic quantum dot, dimensional analysis applied to the $\xi^{-1 / 2}$ scaling of the potential yields

$$
\int_{A} d \mathbf{r} \Psi_{n}^{*}(\mathbf{r}) \Psi_{m}(\mathbf{r}) V_{\mathrm{TF}}(\mathbf{r}) \propto\left(E_{n}-E_{m}\right)^{-1 / 2} .
$$

Such a classical estimation of the matrix elements can be carried out exactly for the case of a diso ${ }^{24}$ or a rectangular strip 25 unbounded in one direction. As in those cases, we expect the scaling in Eq. (19) to be valid also for

\begin{tabular}{||l|c|c||}
\hline \hline$\left.\right|^{\lambda_{\mathrm{TF}} / L}$ & $\gg 1$ & $\ll 1$ \\
\hline$\gg 1$ & dipole, NFL (III) & screened dipole, MFL (V) \\
\hline$\ll 1$ & short range, FL (IV) & short range, FL (IV) \\
\hline \hline
\end{tabular}

TABLE I: Sketch of the regimes studied in the text. FL stands for Fermi liquid behavior, while NFL and MFL indicate nonFermi liquid and marginal Fermi liquid, respectively. The roman numbers indicate the sections where the various cases are discussed in the main text.

any ballistic chaotic quantum dot. This behavior of the matrix element leads to a lifetime:

$$
\Gamma(E) \propto E-E_{F},
$$

i.e., the lifetime of a quasiparticle is proportional to the energy of the quasiparticle itself. This type of dependence is usually associated to marginal Fermi liquid behavior in bulk systems.

Using the results in the Appendix, a more quantitative estimate yields

$$
\Gamma(E) \approx \frac{\lambda_{\mathrm{TF}}^{2}}{z^{d}} \frac{E-E_{F}}{\left(k_{F}^{\prime}\right)^{d-2} k_{F} l^{\prime}} .
$$

Like in the unscreened dipole case, interference among many trajectories leads to $\Gamma(E) \simeq 0$ for $\left(E-E_{F}\right) \ll$ $\hbar v_{F} / L$.

\section{DISCUSSION.}

We have calculated the lifetime of electronic states in ballistic quantum dots due to the presence of metallic gates. Depending on the size of the dot $(L)$, the dotgate distance $(z)$, and the screening length within the dot $\left(\lambda_{\mathrm{TF}}\right)$, we find different regimes, schematically described in Table I. The level width depends quadratically on the quasiparticle energies when the screening is short ranged. This happens when the dot is sufficiently close to the metallic gate or for large dots. Such behavior implies the existence of well defined quasiparticles inside the dot, consistently with the Fermi liquid theory.

We find deviations from this behavior when the dot is sufficiently far from the gate, so that the charge fluctuations at the gate induce an almost uniform electric field at the dot. This field may be either unscreened $\left(L \ll \lambda_{\mathrm{TF}}\right)$ or imperfectly screened $\left(\lambda_{\mathrm{TF}} \ll L\right)$, because of the two-dimensional nature of the dot here considered. In the absence of screening, the coupling between the electrons in the dot and the external gates is correctly described by the standard Caldeira-Leggett model of a particle coupled linearly in its position to a bath of oscillators. The lifetime then shows a plateau at energies $E-E_{F}$ smaller than $E_{F}$, but still larger than $\hbar v_{F} / L$ (see Fig. (31)). This corresponds to non-Fermi-liquid behavior. When the dipole induced by the gate is (imperfectly) screened, the level width is linear in the energy. Then the 
definition of the quasiparticle peaks is not enhanced near the Fermi energy as strongly as in the case of a Fermi liquid. This is the case of a marginal Fermi liquid.

Remarkably, the effect of the gate is enhanced for gates of reduced dimensions, as revealed by the $z^{-d}$ dependence of the linewidths. In this sense, it is important to note that the effective dimensionality $d$ of the response of the gate is determined by the ratio of its various dimensions to the distance to the dot. For instance, the potential fluctuations induced at a distance $z$ by a metallic wire of diameter much smaller than $z$ can be described as if the wire were one-dimensional (see the Appendix), even if the wire is fully three-dimensional, with its diameter much larger than its Fermi wave length.

Inspection of Table I suggests that the degree of departure from Fermi liquid (FL) behavior seems to go through a maximum as a function of the dot-gate distance: At small distances, short-range correlations dominate causing FL behavior to prevail, as shown in section IV. This limit is essentially equivalent to that in which the dot merges into the gate, with global FL behavior. At longer distances, the analysis of sections III and V indicates a departure from the FL regime, especially for small dots. At much longer distances, one expects the interaction between dot and gate to be negligible. Then internal, shortrange correlations dominate within the dot and FL properties are again recovered. We note that this apparently reentrant behavior is compatible with the monotonous decay of the electronic linewidth with distance [See. Eq. [4, since the prefactor of the quadratic energy dependence characteristic of FL behavior are very large in the case of short distances.

It is interesting to compare the estimates obtained here with the lifetimes expected from the decay of the quasiparticles into internal excitations of the dot. That calculation, for a disordered dot, gives 1 :

$$
\Gamma(E) \simeq \frac{1}{k_{F} \lambda_{\mathrm{TF}}} \frac{\left(E-E_{F}\right)^{2}}{E_{F}} .
$$

Comparing this expression with the contribution from the gate, Eq. (21) (we assume $L \gg \lambda_{\mathrm{TF}}$, i.e., the screened case), we find that the effect of the gate dominates if

$$
E-E_{F}<\frac{\hbar v_{F}}{L}\left(\frac{L}{z}\right)^{d} \frac{\left(k_{F} \lambda_{\mathrm{TF}}\right)^{3}\left(k_{F}^{\prime} L\right)^{2-d}}{\left(k_{F} l^{\prime}\right)\left(k_{F} L\right)} .
$$

This inequality is based on the result (21), which only applies for $\left(E-E_{F}\right)>\hbar v_{F} / L$, being zero below that energy. Thus, the dominance of gate effects over a significant energy range requires:

$$
\left(k_{F}^{\prime} z\right)^{d}<\frac{L}{l^{\prime}} \frac{\left(k_{F} \lambda_{\mathrm{TF}}\right)^{3}}{\left(k_{F} / k_{F}^{\prime}\right)^{2}} .
$$

Since, in turn, the dipole approximation requires $z \gg L$, one is led to the condition:

$$
\begin{array}{ll}
k_{F}^{\prime} L \ll\left[\frac{\left(k_{F}^{\prime} / k_{F}\right)^{2}}{k_{F}^{\prime} l^{\prime}}\left(k_{F} \lambda_{\mathrm{TF}}\right)^{3}\right]^{1 /(d-1)} & d>1 \\
1 \ll \frac{\left(k_{F}^{\prime} / k_{F}\right)^{2}}{k_{F}^{\prime} l^{\prime}}\left(k_{F} \lambda_{\mathrm{TF}}\right)^{3} & d=1
\end{array}
$$

Since the quantity with square brackets is $\sim 10^{2}$, we conclude that the observation of the effects discussed here is entirely possible if the gate is effectively one-dimensional ( $z \ll W$, where $W$ is the width of the wire). The optimal range of distance is, roughly, $L<z<100 L$. On the other hand, the observation would be difficult for a two-dimensional gate, since it would require $k_{F}^{\prime} L<10^{2}$, and practically impossible for a semi-infinite $3 \mathrm{D}$ gate.

The unscreened regime, $\lambda_{\mathrm{TF}} \gg L$, corresponds to dots smaller than $\hbar^{2} \epsilon_{0} / m^{*} e^{2}$, which, for realistic values of the parameters, $\epsilon_{0} \sim 12, m^{*} \sim 0.06 m_{e}$, corresponds to $L<$ $10^{2} \AA$. In addition, the calculations presented here require the number of electrons in the dot to be much larger than one. These dots require electronic densities of order $\sim$ $10^{14} \mathrm{~cm}^{-2}$. This value is much higher than those achieved with present day techniques.

For sufficiently large dots, the mean free path $l$ becomes smaller than the size $L$, and we have a crossover from the ballistic regime considered here to a diffusive one. In diffusive systems, typical matrix elements involving states $|m\rangle$ and $|n\rangle$ do not depend on the energy difference $E_{n}-E_{m}$ for energies such that $\left|E_{n}-E_{m}\right| \ll E_{c}$, where $E_{c}=\hbar D / L^{2}$ is the Thouless energy. This corresponds to an effective short-range interaction behavior, as discussed in Sec. IV [see Eq. [15)]. Thus, in the disordered regime, the anomalous effects discussed in this paper will be cut off at energies of order $E_{c}$, i.e., we will have conventional Fermi liquid lifetimes for $E-E_{F}<E_{c}$.

The small sizes and the two-dimensional character of the quantum dot are essential for the non-Fermi liquid results that we have obtained. A three dimensional dot with $\lambda_{\mathrm{TF}}<L$ would result in a complete screening of the gate fluctuations, putting us in the short range regime and thus yielding a Fermi-liquid type of quasiparticle lifetime. For one-dimensional systems, the decay rate of a single particle level of quantum number $n$, coupled to a Caldeira-Leggett environment, has been shown ${ }^{23}$ to scale linearly with $n$. The naive extension of such a universal behavior for fermionic systems would also lead to an electronic lifetime that exhibits a plateau as a function of $E-E_{F}$. However, our starting point of well defined quasiparticles would not be appropriate in one dimension.

Finally, we wish to stress that, due to the reciprocal character of the microscopic interactions, in those cases where have found a departure from Fermi liquid behavior in the dot, a similar deviation will be realized locally in the region of the metallic gate which is most strongly affected by the presence of the quantum dot.

Possible experiments to measure the electronic lifetimes here studied may include transport spectroscopy of quantum dots ${ }^{31.32}$ or, through their effect on the pattern of standing waves, STM studies of the electron density within the dot similar to those performed for quantum corrals 33,34 . 


\section{ACKNOWLEDGMENTS.}

We are grateful to G.-L. Ingold and F. von Oppen for useful discussions. This work has been supported by the EU TMR Program under Contract No. HPRN-CT-200000144. The support of the Ministerio de Ciencia y Tecnología (Spain) under Grants No. BFM2001-0172 and MAT2002-0495-C02-01 is also acknowledged.

\section{APPENDIX A: DIPOLAR COUPLING TO A METALLIC GATE.}

In this appendix we estimate the coefficient $S_{0}$ defining the structure factor in the dipole approximation. For specificity, we will assimilate the metallic gate to a disordered electron layer. The function $S\left(\mathbf{r}, \mathbf{r}^{\prime} ; E\right)$ defined in Eq. (5), where $\mathbf{r}$ and $\mathbf{r}^{\prime}$ are positions within the dot, can be written, in general, as

$$
S\left(\mathbf{r}, \mathbf{r}^{\prime}, z ; E\right)=-\operatorname{Im} V_{\mathrm{scr}}\left(\mathbf{r}-\mathbf{r}^{\prime}, z ; E / \hbar\right)
$$

where $V_{\text {scr }}$ is the screened interaction induced by the gate. For a two dimensional gate located at a distance $z$ from the dot, the Fourier transform of $V_{\text {scr }}$ is:

$$
V_{\mathrm{scr}}(\mathbf{q}, z ; \omega)=\frac{2 \pi e^{2} e^{-2 q z}}{q \epsilon(\mathbf{q}, \omega)}
$$

where $\mathbf{q}$ is a two-dimensional vector in the plane of the dot. For a diffusive $2 \mathrm{D}$ electron gas, we have:

$$
\epsilon(\mathbf{q}, \omega)=1+\frac{2 \pi e^{2}}{q} \frac{D \nu_{2} q^{2}}{D q^{2}-i \omega}
$$

where $D=\hbar^{2} k_{F}^{\prime} / m l^{\prime}$ is the diffusion coefficient, $l^{\prime}$ the mean free path, and $\nu_{2}=m / \pi \hbar^{2}$ is the density of states of the two-dimensional gate. In the long wavelength limit, we obtain

$$
\operatorname{Im} V_{\mathrm{scr}}(\mathbf{q}, z ; \omega) \simeq-\frac{e^{-2 q z} \omega}{D \nu_{2} q^{2}}
$$

where we have assumed $\omega \ll D q^{2}$. The exponential $\exp (-2 q z)$ implies that only wave-vectors such that $q \ll$ $z^{-1}$ contribute to $V_{\mathrm{scr}}\left(\mathbf{r}-\mathbf{r}^{\prime}, z ; \omega\right)$. Assuming that the typical distances within the dot are such that $\left|\mathbf{r}-\mathbf{r}^{\prime}\right| \ll z$, we can write:

$$
\operatorname{Im} V_{\mathrm{scr}}\left(\mathbf{r}-\mathbf{r}^{\prime}, z ; \omega\right) \simeq-\int \frac{d^{2} q}{8 \pi^{2}} \frac{\left|\mathbf{q} \cdot\left(\mathbf{r}-\mathbf{r}^{\prime}\right)\right|^{2} e^{-2 q z} \omega}{D \nu_{2} q^{2}}
$$

which leads to the dipolar approximation discussed in the text ${ }^{35}$.

The calculation of $\operatorname{Im} V_{\mathrm{scr}}\left(\mathbf{r}-\mathbf{r}^{\prime}, z ; \omega\right)$ for a semi-infinite three-dimensional gate can be carried out in a similar way if we assume that quasiparticles within the gate are specularly reflected at the boundary ${ }^{36}$. This approximation has been widely used in the literature for the study of the related problems of energy dissipation by moving charges 37 or the decay of image states at metallic surfaces 38.39 . Then, the effective dielectric function which describes the effect of electrostatic screening by the gate at points outside the gate can be written as a twodimensional integral over the surface of the gate. This approximation becomes exact at large distances. The resulting screened potential outside the gate is:

$$
V_{\text {scr }}(\mathbf{q}, z ; \omega)=e^{-2 q z} \frac{2 \pi e^{2}}{q} \frac{B(\mathbf{q}, \omega)-1}{B(\mathbf{q}, \omega)+1},
$$

where

$$
B(\mathbf{q}, \omega) \equiv \frac{q}{\pi} \int \frac{d q_{z}}{\left(q^{2}+q_{z}^{2}\right) \epsilon\left(\mathbf{q}, q_{z} ; \omega\right)}
$$

$\epsilon\left(\mathbf{q}, q_{z}, \omega\right)$ being the bulk dielectric function. Using, as for the two-dimensional gate, the dielectric function of a diffusive electron liquid, and expanding for $\left|\mathbf{r}-\mathbf{r}^{\prime}\right| \ll$ $z$, we find a result identical to Eq. A5 with $\nu_{2} q^{2}$ in the denominator of the integrand replaced by $\nu_{3} q$, where $\nu_{3}=m k_{F}^{\prime} / \pi^{2} \hbar^{2}$ is the density of states of the threedimensional gate.

Finally, it is straightforward to generalize the previous calculations to the case where the gate is a onedimensional metallic gate. The main difference is that the integral over the positions at the gate is onedimensional.

Using eqs. A5 and A6 we can estimate the value of $S_{0}$ in Eq. (9). We find, apart from factors of order unity,

$$
S_{0}^{-1}(z) \approx \begin{cases}z l^{\prime} & 1 \mathrm{D} \\ z^{2}\left(k_{F}^{\prime} l^{\prime}\right) & 2 \mathrm{D} \\ z^{2}\left(k_{F}^{\prime} z\right)\left(k_{F}^{\prime} l^{\prime}\right) & 3 \mathrm{D}\end{cases}
$$

Corrections of order $\log (z / R)$, where $R$ is the radius of the gate, are neglected when the gate is effectively onedimensional.
1 U. Sivan, Y. Imry, and A. G. Aronov, Europhys. Lett. 28, 115 (1994).

${ }^{2}$ Y. Imry, A. Stern, and U. Sivan, Europhys. Lett. 39, 639
(1997).

3 B. L. Altshuler, Y. Gefen, A. Kamenev, and L. S. Levitov, Phys. Rev. Lett. 78, 2803 (1997). 
${ }^{4}$ P. G. Silvestrov, Phys. Rev. Lett. 79, 3994 (1997).

5 P. Nozières, The Theory of Quantum Liquids, AddisonWesley, New York (1989).

6 T. Ando, A. B. Fowler, and F. Stern, Rev. Mod. Phys. 54, 437 (1982).

7 Y. Alhassid, Rev. Mod. Phys. 72, 895 (2000).

8 I. L. Aleiner, P. W. Brouwer, and L. I. Glazman, Phys. Rep. 358, 309 (2002).

9 L.P. Kouwenhoven, C.M. Marcus, P.L. McEuen, S. Tarucha, R.M. Westervelt, and N.S. Wingreen, in Proc. NATO-ASI on Mesoscopic Electron Transport, edited by L.L. Sohn, L.P. Kouwenhoven, and G. Schön (Kluwer Series E345) 105 (1997).

10 L.P. Kouwenhoven, D.G. Austing, and S. Tarucha, Rep. Prog. Phys. 64, 701 (2001).

11 U. Weiss, Quantum dissipative systems (World Scientific, Singapore, 1993).

12 A. O. Caldeira and A. J. Leggett, Phys. Rev. Lett. 46, 211 (1981).

13 P. Cedraschi, M. Büttiker, Ann. Phys. (New York) 289, 1 (2001).

14 A. Yacoby, M. Heiblum, D. Mahalu, and H. Shtrikman, Phys. Rev. Lett. 74, 4047 (1995).

15 R. M. Clarke et al., Phys. Rev. B 52, 2656 (1995).

16 E. Buks, R. Schuster, M. Heiblum, D. Mahalu and V. Umanksy, Nature 391, 871 (1998).

17 J. A. Folk, C. M. Marcus, J. S. Harris, Jr., Phys. Rev. Lett. 87, 206802 (2001).

18 M. Feingold and A. Peres, Phys. Rev. A 34, 591 (1986).

19 M. Wilkinson, J. Phys. A 20, 2415 (1987).

20 B. Mehlig, D. Boosé, and K. Müller, Phys. Rev. Lett. 75, 57 (1995); B. Mehlig, Phys. Rev. E 59, 390 (1999).

21 B. Eckardt, S. Fishman, K. Müller, and D. Wintgen, Phys. Rev. A 45, 3531 (1992).

22 M. C. Gutzwiller, Chaos in Classical and Quantum Mechanics (Springer-Verlag, NY, 1990).
${ }^{23}$ G.-L. Ingold, R. A. Jalabert, and K. Richter, Am. J. Phys. 69, 201 (2001).

24 E. J. Austin, and M. Wilkinson, J. Phys.: Condens. Matter 5, 8461 (1993)

25 M. Santer, and B. Mehlig, Phys. Rev. B 63, 241403 (2001).

${ }^{26}$ K. Richter, Semiclassical Theory of Mesoscopic Quantum Systems, Springer Trac. Mod. Phys. (Springer-Verlag, Berlin, 2000).

27 D. S. Golubev and A. Zaikin, Physica B 255, 164 (1998).

28 F. Guinea, Phys. Rev. B 65, 205317 (2002); ibid. 67, 045103 (2003).

29 A. D. Mirlin, Phys. Rep. 326, 259 (2000).

${ }^{30}$ P. A. Lee, and T. V. Ramakrishnan, Rev. Mod. Phys. 57, 287 (1985).

31 T. Ihn, A. Fuhrer, T. Heinzel, K. Ensslin, W.Wegscheider, and M. Bichler, Physica E 16, 83 (2003).

32 M. Sigrist, A. Fuhrer, T. Ihn, E. Ensslin, S. E. Ulloa, W.Wegscheider, and M. Bichler, cond-mat/0308223 to be published.

33 H. C. Manoharan, C. P. Lutz, and D. M. Eigler, Nature 403, 512 (2000).

${ }^{34}$ G. A. Fiete and E. J. Heller, Rev. Mod. Phys. 75, 933 (2003).

35 The factor $\left|\mathbf{q} \cdot\left(\mathbf{r}-\mathbf{r}^{\prime}\right)\right|^{2}$ in the integrand is equivalent to $q^{2}\left(r^{2}+r^{\prime 2}-2 \mathbf{r} \cdot \mathbf{r}^{\prime}\right) / 2$. Since the term $\left(r^{2}+r^{\prime 2}\right)$ does not contribute to the decay rate in Eq. (2), we obtain effectively the bilinear behavior $S\left(\mathbf{r}, \mathbf{r}^{\prime}\right) \sim \mathbf{r} \cdot \mathbf{r}^{\prime}$ which characterizes the coupling for $z \gg L$.

36 R. H. Ritchie, and A. L. Marusak, Surf. Sci. 4, 234 (1966).

37 F. Sols and R. H. Ritchie, Surf. Sci. 194, 275 (1988).

38 P. M. Echenique, F. Flores, and F. Sols, Phys. Rev. Lett. 55, 2348 (1985).

39 P. M. Echenique, J. M. Pitarke, E. V. Chulkov, and A. Rubio, Chem. Phys. 251, 1 (2000). 